\title{
PROCESS AND SYSTEMS The impact of an acute chest pain pathway on the investigation and management of cardiac chest pain
}

\author{
Authors: Mark Sweeney, ${ }^{\mathrm{A}}$ Gillian Bleeze, ${ }^{\mathrm{B}}$ Sarah Storey, ${ }^{\mathrm{C}}$ Alexander Cairns, ${ }^{\mathrm{D}}$ Alexander Taylor, ${ }^{\mathrm{E}}$ Catherine Holmes, ${ }^{\mathrm{F}}$ \\ Steve Hart, ${ }^{G}$ Nick Lawrance, ${ }^{H}$ William G Oldfield ${ }^{\mathrm{I}}$ and Christopher S Baker
}

\begin{abstract}
Emergency department (ED) presentation with chest pain accounts for approximately $20 \%$ of acute hospital admissions, and delays in the investigation and management of these patients increase the pressure on emergency and medical departments. We implemented a pathway within our trust to improve the efficiency of acute chest pain management. This included the development of a chest pain management algorithm, a short-stay heart assessment centre and a policy to immediately transfer acute coronary syndrome patients to cardiology. The introduction of the chest pain pathway resulted in fewer admissions from the ED with chest pain ( $34.2 \%$ vs $19.0 \%$; $\mathrm{p}<0.0001$ ), a reduction in time from ED attendance to cardiology transfer $(9.3$ hours vs 5.7 hours; $p<0.0001)$ and $a$ reduction in time to angiography ( 62.5 hours vs 26.6 hours; $\mathrm{p}<0.0001)$. Length of stay was reduced for cardiology patients ( 4.7 days vs 2.4 days, $p<0.001$ ) and mean length of stay for all patients attending ED with chest pain was reduced by 8.3 hours ( 27.5 hours vs 19.1 hours; $p<0.0001$ ). The changes have significantly improved the management of acute chest pain within our trust and we would suggest that adoption of these changes in other trusts could significantly improve the quality of the care for these patients throughout the NHS.
\end{abstract}

KEYWORDS: Chest pain, acute coronary syndrome, service improvement

Authors: ${ }^{\mathrm{A}}$ National Institute of Health Research academic clinical fellow, Imperial College Healthcare NHS Trust, London, UK and London Institute of Medical Science, London, UK; ${ }^{B}$ senior nurse cardiac service, Imperial College Healthcare NHS Trust, London, UK; ' matron inpatient cardiology, Imperial College Healthcare NHS Trust, London, UK; ${ }^{D}$ foundation year-1 doctor, Imperial College Healthcare NHS Trust, London, UK; Efoundation year-2 doctor, Imperial College Healthcare NHS Trust, London, UK; F transformation programme director, Royal Devon and Exeter NHS Trust, Exeter, UK; ' general manager circulation sciences, Imperial College Healthcare NHS Trust, London, UK; ${ }^{H}$ transformation lead, Imperial College Healthcare NHS Trust, London, UK; ' deputy medical director, Imperial College Healthcare NHS Trust, London, UK; ' consultant cardiologist, Imperial College Healthcare NHS Trust, London, UK

\section{Introduction}

Chest pain is one of the most common reasons for presentation to the emergency department (ED), accounting for $2-6 \%$ of ED attendances and $20 \%$ of emergency admissions to hospital. ${ }^{1-4}$ Most of these admissions are to perform risk assessment for a potential diagnosis of acute coronary syndrome (ACS) which requires measurement of cardiac biomarkers. ${ }^{5}$

Patients within London presenting with chest pain who fulfil the primary angioplasty or high-risk ACS criteria (Box 1) are taken directly to a heart attack centre. However, patients who do not meet these emergency criteria undergo further assessment and risk stratification within the nearest ED.

Our institution is a tertiary trust based across five sites in west London, the two EDs in Charing Cross Hospital and St Mary's Hospital are located approximately 3 miles away from the cardiac centre in Hammersmith Hospital. This geographical separation is common for multiple cardiac centres throughout the UK.

Delays in treating and discharging patients with low/ intermediate risk ACS led the trust in 2016 to devise and implement a dedicated chest pain pathway with the intention of avoiding unnecessary admission with chest pain and reducing time spent awaiting cardiology transfers and cardiac

Box 1. Emergency heart assessment centre transfer criteria from ambulance service

\section{Ongoing chest pain}

plus

\section{Primary angioplasty call}

$>\mathrm{ST}$ segment elevation $>1 \mathrm{~mm}$ in two contiguous limb leads.

$>$ ST segment elevation $>2 \mathrm{~mm}$ in two contiguous chest leads.

$>$ Left bundle branch block or paced rhythm if the clinical picture is acute myocardial infarction.

> Patients resuscitated from cardiac arrest with electrocardiography criteria as above.

or

High risk acute coronary syndrome

ST depression $>2 \mathrm{~mm}$ in two or more contiguous leads.

$>$ Deep symmetrical T wave inversions in two or more contiguous leads. 
investigations, therefore improving patient experience and reducing their time spent in hospital.

\section{Existing pathway}

The existing model for managing chest pain involved the measurement of conventional sensitivity troponin at 6 and 12 hours to check for a dynamic rise. Patients with raised serum troponin and/ or dynamic electrocardiography (ECG) changes and a consistent history would be referred to the cardiology registrar on call in the cardiac centre who would accept the patient for transfer to cardiology on a case-by-case basis. Accepted patients were placed on a transfer list to await a bed becoming available on the cardiology ward which could often take a number of days. This model reduced flow from our EDs into inpatient beds at the two ED sites, and feedback from the patient surveys frequently commented on the number of wards they had to visit before arriving at the heart attack centre.

\section{Methods}

\section{The development of the chest pain pathway}

To manage a wider change programme to align Hammersmith Hospital with the vision for it to become a specialist (rather than traditional acute) site, a fortnightly working group was set up with the management of chest pain presenting to the ED as one of their key deliverables. It was led by the deputy medical director with a full-time dedicated project management resource for the design and implementation period. The working group included the divisional director, director of nursing and directors of operations from cardiology and medicine. One weekly programmed activity was funded for clinical lead of the chest pain project and there was extensive input from senior nursing staff, finance and information and communications technology leads, divisional managers, lead clinicians from affected specialities and the ambulance service. The central tenets included:

> implementation of a standardised assessment protocol

> direct and immediate 'no quibble' transfer of patients to a redeveloped heart assessment centre (HAC) at the Hammersmith
$>$ an aim to provide invasive investigation and treatment within 24 hours of arrival to the hospital.

\section{High-sensitivity troponin chest pain algorithm}

A chest pain algorithm was developed to help risk stratify patients presenting with chest pain using the GRACE 1.0 (Global Registry of Acute Coronary Events) score and TIMI (Thrombolysis in Myocardial Infarction) score (Fig 1). High GRACE or TIMI scores identified high risk patients who should be prioritised for early transfer to cardiology and consideration of an early invasive angiographic approach within 24 hours which has been shown to improve outcomes in this high-risk group. ${ }^{6,7}$

Advances in high-sensitivity troponin (hs-troponin) assays has enabled myocardial damage to be detected earlier following the onset of pain and the most recent European Society of Cardiology (ESC) and National Institute of Health and Care Excellence (NICE) guidance have changed to reflect this. ${ }^{8-13}$ Our institution used the NICE recommended Abbott Architect STAT troponin-I with sex-specific cut-offs. ${ }^{13,14}$ Recent studies have shown that patients with undetectable hs-troponin levels at presentation are at low risk of future cardiac events. ${ }^{11,15,16}$ Therefore, patients who are pain free with a normal resting ECG and undetectable hs-troponin-I measured at least 120 minutes after onset of chest pain can be reassured and discharged from ED with appropriate follow-up., ${ }^{9,15}$ Patients with highly abnormal hs-troponin-I results at admission (male > $>68 \mathrm{ng} / \mathrm{L}$; female $>30 \mathrm{ng} / \mathrm{L}$ ) should be referred to cardiology on the chest pain pathway as soon as this result is available. An initial troponin measurement under the high-risk threshold should be repeated after 3 hours to look for an interval change of greater than $50 \%$ or an absolute value $>99$ th percentile which should prompt referral to the cardiology registrar on the chest pain pathway.

\section{Immediate transfer}

Patients who meet the chest pain pathway criteria are referred to the cardiology registrar and transferred immediately to the cardiology centre for further review and investigation. These

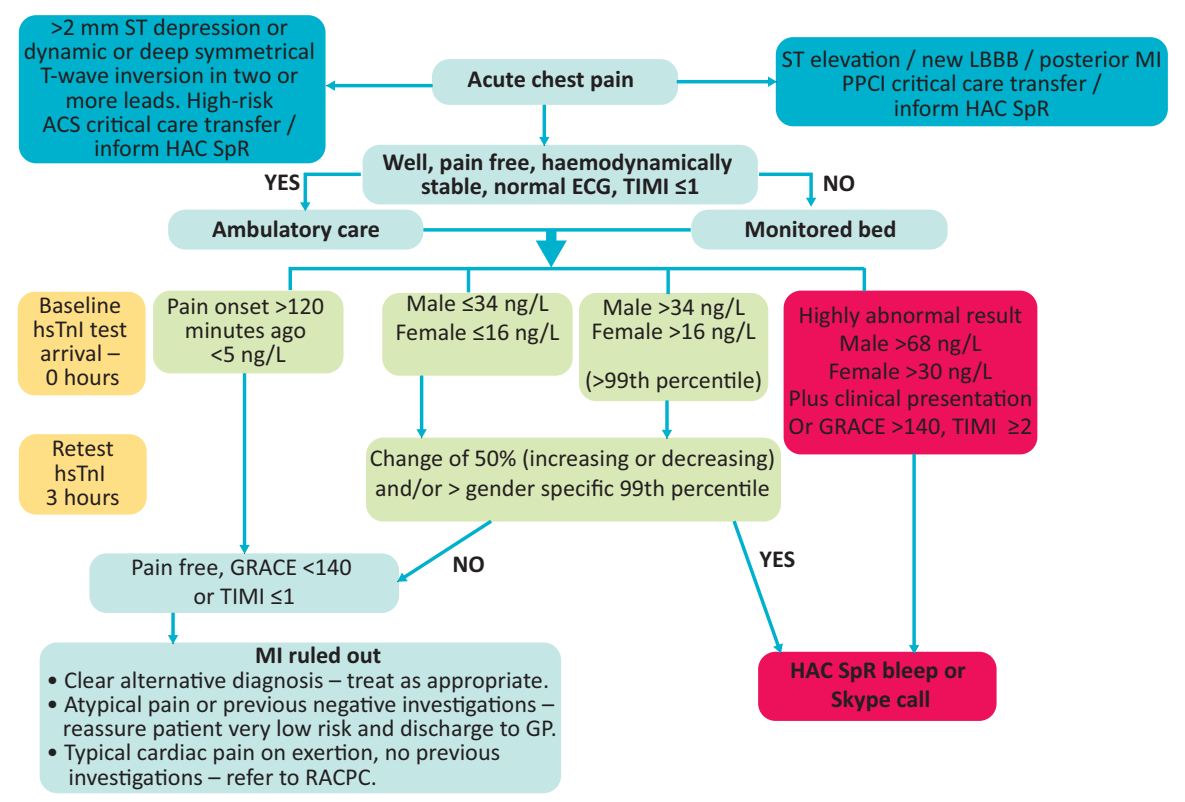

Fig 1. Chest pain algorithm implemented throughout the trust to guide the investigation and management of all patients presenting to the emergency department with chest pain. ACS = acute coronary syndrome; ECG = electrocardiography; GP = general practitioner; GRACE = Global Registry of Acute Coronary Events score; $\mathrm{HAC}=$ heart assessment centre; hsTnI = high-sensitivity troponin-I; LBBB $=$ left bundle branch block; PPCI = primary percutaneous coronary intervention; RACPC $=$ rapid access chest pain clinic; $S p R=$ specialist registrar; TIMI $=$ Thrombolysis in Myocardial Infarction score. 
transfers occur regardless of the cardiology bed state at the time and the cardiology team are responsible for ensuring beds are available for chest pain pathway patients on transfer. There is a dedicated cardiology bed manager to facilitate this which would involve the opening of escalation beds if necessary.

\section{Heart assessment centre}

To facilitate the increased number and acuity of patients which accompanied this change in practice, the short-stay HAC was redeveloped. This became a 9-bed unit which was designed to admit patients for less than 24 hours to allow cardiology assessment, angiography and angioplasty if required, and recovery following these procedures. The HAC included the addition of three new assessment bays with a dedicated anaesthetic machine for emergencies and an adjoining room equipped with an echocardiography machine and couch. Weekend nursing cover was increased so that the unit is staffed 7 days per week by three cardiac trained nurses and one healthcare assistant. Medical cover is provided by a dedicated cardiology registrar and senior house officer during the day and the on-call cardiology team overnight. A Saturday inpatient angioplasty list was introduced which prevented patients waiting over the weekend for procedures and dedicated 7 day per week HAC echocardiography service was set up to avoid delays in discharge for patients awaiting echocardiography.

There is a daily consultant morning ward round to assess all overnight admissions to ensure clear plans for further investigation, treatment and discharge are made for all patients. Admissions during the day are discussed with consultants covering the cardiac catheter labs with the aim to perform angiography on the same day.

\section{Cardiology medicine for the elderly ward}

The majority of patients admitted with ACS to the HAC are appropriate for discharge directly from the HAC following angiography or angioplasty. However, elderly patients or those with significant comorbidities or complex social needs are often not suitable for an accelerated discharge. As part of the process we created a 15-bed cardiology step-down ward where patients with complex medical and social needs can be transferred following the completion of their cardiology investigations and treatment. This unit has intensive input from occupational therapy and physiotherapy and daily ward rounds by a consultant geriatrician. They aim to optimise management of comorbidities and identify complex discharge issues which can be preemptively addressed to minimise delays during discharge and improve the quality of discharge to prevent readmissions.

\section{Analysis}

To assess the impact of the new chest pain pathway on our service we retrospectively analysed all patients attending the ED in our institution with a triage diagnosis of chest pain, before and after the implementation of the chest pain pathway. This included accessing electronic patient notes, angiography records, and admission, transfer and discharge times from the electronic hospital system. Timing of transfer to the wards from ED or between wards within the trust was captured when they are moved on the electronic system. Discharge times are recorded electronically by the ward administrator when the patient leaves the ward, or by the nurse in charge if this occurs out of hours or from ED. We collected data for a period of 8 months prior to the introduction of any changes and 18 months after the completion of the introduction of the chest pain pathway.

\section{Statistics}

Statistical analysis was performed in SPSS (IBM SPSS Statistics v25) and GraphPad Prism (GraphPad Software v8.0, San Diego, USA). Results are reported as median \pm interquartile range unless otherwise stated. Comparisons between groups are reported as baseline vs post implementation of the chest pain pathway unless stated otherwise. $\chi^{2}$ test of independence was used to analyse categorical data before and after intervention and Mann-Whitney $U$ test was used to analyse continuous data, including changes in transfer time, time to angiography and length of stay.

\section{Results}

During the study period, there were 21,377 attendances to the EDs with a triage diagnosis of chest pain, equating to approximately 10,000 patients per year across the trust (Table 1).

\section{Reduction in emergency department admissions}

The proportions of patients requiring admission from the ED was reduced significantly following the introduction of the pathway (34.2\% vs $19.0 \%$; $p<0.0001$; Fig 2). This included reductions in short term admissions to the clinical decisions unit (CDU; $10.5 \%$ vs $5.7 \% ; p<0.0001)$ and admission to the medical wards ( $22.8 \%$ vs $9.6 \% ; p<0.0001$; Table 2).

\section{Reattendance}

The reattendance rate following discharge from the ED showed a small but significant increase at 30 days $(5.8 \%$ vs $7.6 \%$; $p<0.001)$. However, the number of patients who reattended and required cardiology admission on the second attendance was very low in both groups $(0.5 \%$ vs $1.8 \%$; $<<0.05)$ and a very small number of these patients underwent angioplasty on these subsequent admissions ( $0.5 \%$ vs $0.8 \%$; not significant (NS)) suggesting that pathway was not resulting in high-risk chest pain presentations being erroneously discharged. The GRACE score of patients discharged from ED who required cardiology admission on the second attendance was low $(<109)$ in $77 \%$ and intermediate (109-140) in $18 \%$ of cases. There was one case of a patient with a high-risk GRACE score who was discharged and reattended with a non-ST segment

Table 1. Descriptive data of patients attending

emergency department with triage diagnosis of

chest pain during the study period

\begin{tabular}{|c|c|c|}
\hline & Baseline & Chest pain pathway \\
\hline Dates & $\begin{array}{l}\text { April } 2015 \text { - } \\
\text { November } 2015\end{array}$ & $\begin{array}{l}\text { September } 2016 \text { - } \\
\text { February } 2018\end{array}$ \\
\hline Number & 5,495 & 15,882 \\
\hline Mean age, years & $50.3 \pm 14.7$ & $49.9 \pm 14.2$ \\
\hline Age $>65$ years, $n(\%)$ & $1,408(26 \%)$ & $3,839(24 \%)$ \\
\hline $\begin{array}{l}\text { Troponin measured, } \\
\mathrm{n}(\%)\end{array}$ & $3,732(68 \%)$ & $12,663(80.0 \%)$ \\
\hline
\end{tabular}




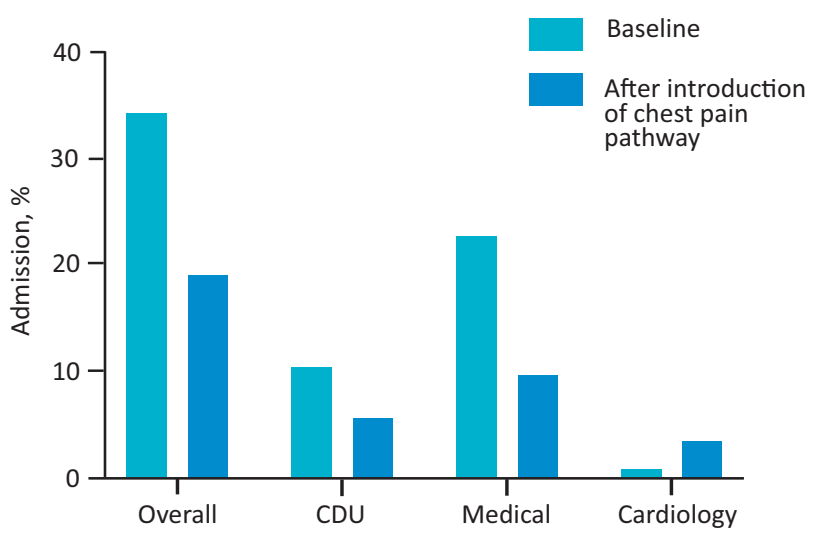

Fig 2. Percentage of total attendances to the emergency department with a triage diagnosis of chest pain that were admitted to the hospital, clinical decisions unit, medical wards and directly to the cardiology ward before and after the introduction of the chest pain pathway. For all changes after introduction $p<0.0001$. CDU $=$ clinical decisions unit.

elevation myocardial infarction 20 days later, however did not undergo angiography during that admission due to frailty.

\section{Reduction in time to cardiology transfer and angiography}

The median wait in hospital prior to transfer to the cardiology centre was reduced by the chest pain pathway $(14.5 \pm 50.3$ hours vs $5.7 \pm 6$ hours; $p<0.0001$ ). Within 12 hours of attending $E D, 82 \%$ of patients who required inpatient cardiology admission had been transferred to a cardiology ward compared to $46 \%$ of patients before the pathway was introduced (Fig 3a).

The median time to angiography was also significantly reduced $(62.5 \pm 72.4$ hours vs $28.6 \pm 32.4$ hours; $p<0.0001$; Fig $3 b)$ and the proportion of patients who had angiography performed within 24 hours of arrival to ED improved from $12 \%$ to $40 \%$.

\section{Patient experience}

The results of a 13-question patient discharge survey after the implementation of the chest pain pathway demonstrated an excellent degree of satisfaction with an overall experience score of $96 \%$. Ninety-eight per cent of patients said they would be happy to recommend the service to a friend or family member in the same situation. It was not possible to retrospectively collect data regarding the patient experience before the chest pain pathway as there was no dedicated chest pain admission pathway and these patients were grouped with all cardiology admissions. However, one of the recurring complaints noted on patient surveys prior to the introduction of the chest pain pathway was multiple ward transfers which occurred before arriving on a cardiac ward. Twentytwo per cent of patients were admitted to at least two medical wards prior to arriving with cardiology therefore experiencing a minimum of three ward transfers. After the introduction of the chest pain pathway this was significantly reduced to $8 \%$ $(p<0.0001)$ with $79 \%$ of patients being admitted directly to cardiology from ED compared to $28 \%$ before the pathway $(p<0.0001)$.
Table 2. Descriptive data at baseline and following

the introduction of the chest pain pathway

\begin{tabular}{|c|c|c|}
\hline & Baseline & $\begin{array}{l}\text { Chest pain } \\
\text { pathway }\end{array}$ \\
\hline \multicolumn{3}{|l|}{ Admissions } \\
\hline Admission from ED, $\mathrm{n}(\%)$ & $1,882(34.2)$ & $3,022(19.0)$ \\
\hline \multicolumn{3}{|l|}{ Admission ward } \\
\hline Clinic decision unit, \% & 30.6 & 30.0 \\
\hline Medical bed, \% & 66.6 & 50.5 \\
\hline Cardiology bed, \% & 2.8 & 19.5 \\
\hline $\begin{array}{l}\text { Time to admission, median } \\
\text { hours } \pm \mathrm{IQR}\end{array}$ & $3.9 \pm 1.6$ & $5.0 \pm 3.8$ \\
\hline \multicolumn{3}{|l|}{ Transfer to cardiology } \\
\hline $\mathrm{n}$ ( $\%$ of attendances) & $183(3.3)$ & $741(4.7)$ \\
\hline $\begin{array}{l}\text { Time to transfer, median } \\
\text { hours } \pm \text { IQR }\end{array}$ & $9.3 \pm 29.7$ & $5.7 \pm 5.9$ \\
\hline \multicolumn{3}{|l|}{ Angiography } \\
\hline $\mathrm{n}$ ( $\%$ of attendances) & $120(2.2)$ & $386(2.3)$ \\
\hline $\begin{array}{l}\text { Time to angiography, median } \\
\text { hours } \pm \text { IQR }\end{array}$ & $62.5 \pm 54.2$ & $26.6 \pm 32.8$ \\
\hline Angiography $<72$ hours, n (\%) & $69(57.5)$ & $311(85.2)$ \\
\hline \multicolumn{3}{|l|}{ Length of stay } \\
\hline $\begin{array}{l}\text { Median all attendances, median } \\
\text { hours } \pm \mathrm{IQR}\end{array}$ & $3.8 \pm 9.7$ & $3.8 \pm 3.2$ \\
\hline $\begin{array}{l}\text { Mean all attendances, mean } \\
\text { hours } \pm \text { SD }\end{array}$ & $27.5 \pm 99.9$ & $19.1 \pm 83.9$ \\
\hline $\begin{array}{l}\text { Cardiology admissions, median } \\
\text { days } \pm \mathrm{IQR}\end{array}$ & $4.7 \pm 6.5$ & $2.4 \pm 2.7$ \\
\hline Angiography, median days $\pm \mathrm{IQR}$ & $5.1 \pm 6.6$ & $2.9 \pm 3.2$ \\
\hline
\end{tabular}

\section{Length of stay}

The length of inpatient admission was reduced by the introduction of the pathway. This included patients who were transferred to cardiology $(4.7 \pm 6.5$ days vs $2.4 \pm 2.7$ days; $p<0.0001)$ and those patients who required coronary angiography during their admission ( $5.1 \pm 6.6$ days vs $2.9 \pm 3.2$ days; $p<0.0001$; Fig $3 c$ ). As more than $50 \%$ of patients both before and after the introduction of the pathway were discharged within 4 hours ( $60 \%$ vs $63 \%$ ), the overall median length of stay remained unchanged at 3.8 hours however the 75th percentile reduced from 12.7 hours to 6.1 hours reflecting a reduction in the length of these short admissions and the overall mean length of stay reduced by 8.4 hours $(27.5 \pm 99.9$ hours vs $19.1 \pm 83.3$ hours; $p<0.0001$ ).

\section{Discussion}

We have shown that the introduction of a standardised approach and a clear pathway for the management of patients presenting with chest pain to our trust has reduced the number of admissions by $44 \%$ and reduced the length of time awaiting 
a

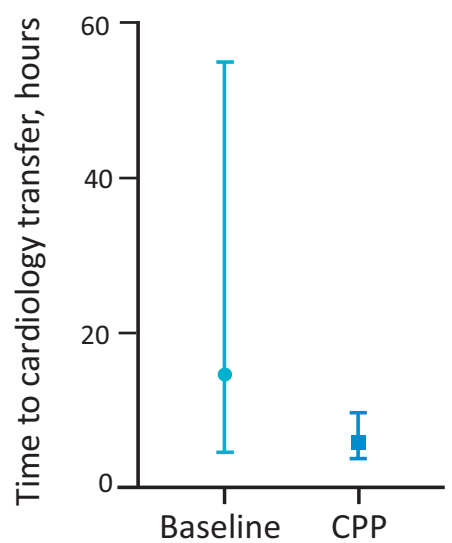

C

Fig 3. a) Time from emergency department attendance with chest pain to cardiology transfer. b) Time from emergency department attendance with chest pain to coronary angiography before and after the introduction of the chest pain pathway. c) Length of stay for all patients admitted to cardiology and those who had a coronary angiography during their admission before (teal) and after (blue) the introduction of the chest pain pathway. d) Overall length of stay for all patients attending emergency department with a triage diagnosis of chest pain. Data represented as median \pm interquartile range. For all changes after introduction $p<0.0001$. CPP $=$ after introduction of chest pain pathway.

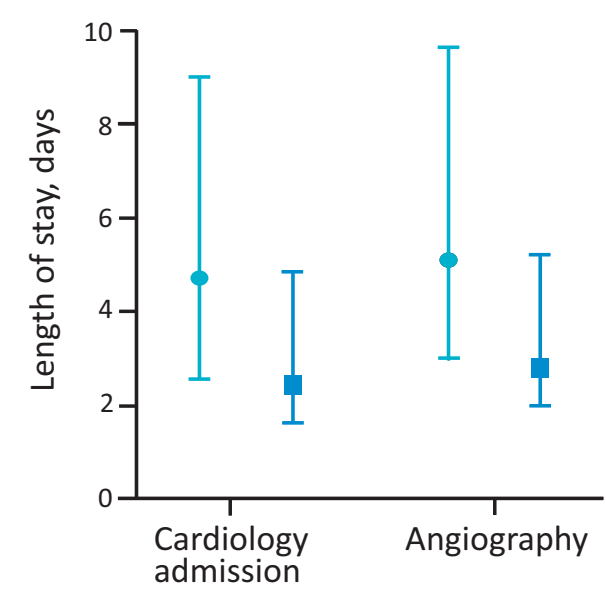

b

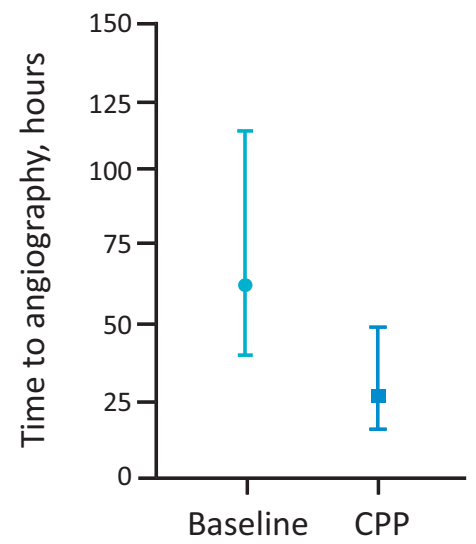

d

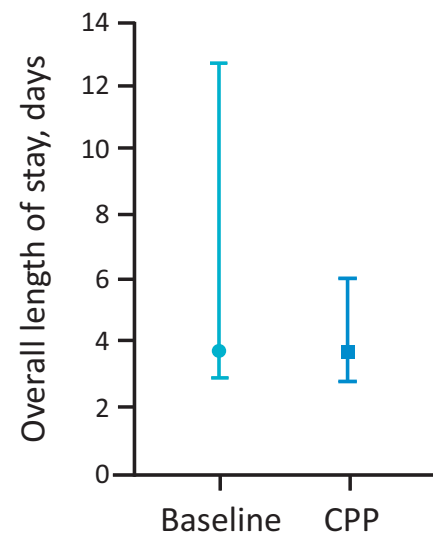

cardiology transfer and angiography. Eighty-five per cent of coronary angiography now occur within the NICE recommended 72-hour timeframe compared to $58 \%$ prior to the chest pain pathway. As a result of these changes, the overall length of stay for patients with chest pain has reduced by 8.4 hours which has saved the trust 3,500 bed-days per year equating to an annual saving of $£ 1.1$ million. $^{17}$

\section{Financial assessment}

Assessment of the cost of implementing this programme was challenging as much of the work was already being undertaken within the trust in different areas and drivers of the changes was not exclusively financial but rather improvements in patient care. The key to realising these improvements was identifying and eliminating unnecessary steps within the pathway and allocating resources to optimise the steps which added value to the patient journey. Initial capital expenditure for the development of the HAC amounted to $£ 242,000$. This included the redevelopment of $\mathrm{HAC}$ and provision of new equipment, including a ventilator for the acute assessment bay, dedicated echocardiography machine and couch, cardiac monitors, resuscitation equipment and improved signage to the HAC. There were increased staffing costs required to cover the increased workload expected within HAC and the coronary labs which included increased weekend nursing cover on HAC ( $£ 33,000$ ), staffing costs for the Saturday angiography list, including a interventional cardiology consultant, radiographer and cardiac physiologist (£117,000) and the increased cost of a sonographer to provide a dedicated HAC echocardiography list ( $£ 99,000$ ). This amounted to a total of $£ 249,000$.

As the cardiology step-down ward was being adapted from an existing medical ward there was little in the way of capital expenditure required. The cost of a consultant geriatrician and nursing costs were now provided from the cardiology budget however on a trust level these patients were already requiring care on medical wards either before or after cardiology care with equivalent staffing costs.

\section{Chest pain algorithm}

The chest pain pathway algorithm includes use of a hs-troponin assay which has been shown to provide a safe and effective rule-out test for the diagnosis of ACS if measured at 0 and 3 hours from presentation. ${ }^{18}$ Additionally meta-analysis data 
has demonstrated that an hs-troponin level below the limit of detection on presentation has a strongly negative predictive valve (NPV) for myocardial infarction (99.3\%). ${ }^{11,19}$ The combination of an hs-troponin level below the level of detection with a validated risk score such as TIMI further increases the NPV. ${ }^{19}$

Importantly, standardisation of the hs-troponin algorithm across the trust has helped the referring teams to focus on the key points of the history and initial investigations which advance the diagnosis and allow early decisions about admission or discharge to be made within the ED. This expedited decision making allowing more patients to be discharged directly from ED or admitted directly to HAC which improves the flow of patients in the department.

By introducing a 'no quibble' policy to automatically accept all patients meeting the chest pain criteria directly to the HAC, we have prevented patients from being admitted unnecessarily under the medical team awaiting transfer. This avoids duplicating the work of admitting and reviewing these patients by the medical team as well as reducing the time dedicated to making phone calls to refer patients and arrange transfers. The resulting reduction in the occupancy of medical beds has improved flow through the emergency and acute medical departments. The capacity of the cardiology unit has been increased through the creation of the HAC to accommodate the increased volume of patients and the introduction of an inpatient Saturday angiography list has increased the availability of coronary angiography and weekend HAC echocardiography lists avoided discharge delays caused by awaiting echocardiography. As a result, patients are able to see the right consultant early, avoid multiple ward transfers, have access to the right investigations and receive optimal treatment as quickly as possible, reducing their time spent in hospital which has been reflected in the excellent patient experience score for this unit.

\section{Limitations}

There is a concern that the removal of cardiology patients from the acute medical take risks negatively impacting medical training as medical trainees are denied exposure to patients with ACS. However, the benefits for patients coupled with the reduction in time and bed pressures on the medical team is likely to be beneficial to training overall.

Although it includes a large number of patients, this analysis is limited by the retrospective nature of the data collection. Additionally, we used the triage diagnosis of chest pain in the inclusion criteria for this analysis. Triage diagnosis is a useful marker to provide a well-defined and reproducible population, however we acknowledge that a significant proportion of patients, particularly women and the elderly, can have ACS presentations without chest pain which will not be included in this analysis. ${ }^{20}$ Two-thirds of the urgent inpatient coronary angiography for ACS in our trust are due to ED presentation with chest pain. Other common presenting symptoms such as breathlessness, collapse and 'unwell adult' make up smaller proportions and are less specific for coronary artery disease. These patients often had significant comorbidities including sepsis, renal failure, heart failure, arrhythmias or valve disease which need to be addressed prior to considering coronary angiography and were often admitted under other specialties in the first instance.

\section{Conclusion}

Despite these limitations, we believe that significant improvements have been generated by the introduction of the chest pain pathway which are clinically meaningful, sustainable and have addressed key issues raised by patients about the quality of their care. Should near patient hs-troponin testing become available, these gains could be improved further with initial assessment being made by the ambulance service. ${ }^{21}$

We would hope that these changes could be used as a template to help implement key improvements in other institutions who are developing a similar service. Although we have implemented this pathway within one trust with a single management structure, the multi-site nature of the project means it should be possible to implement the key design principles across multiple trusts within a region, and the benefits to sites without 24 -hour cardiology cover may be even greater.

\section{References}

1 Fothergill NJ, Hunt MT, Touquet R. Audit of patients with chest pain presenting to an accident and emergency department over a 6-month period. Arch Emerg Med 1993;10:155-60.

2 Goodacre S, Cross E, Arnold ] et al. The health care burden of acute chest pain. Heart 2005;91:229-30.

3 Blatchford O, Capewell S, Murray S, Blatchford M. Emergency medical admissions in Glasgow: general practices vary despite adjustment for age, sex, and deprivation. Br J Gen Pract 1999:49:551-4.

4 MacIntyre K, Murphy NF, Chalmers ] et al. Hospital burden of suspected acute coronary syndromes: recent trends. Heart 2006;92:691-2.

5 DeBusk RF, Miller NH, Raby L. Technical feasibility of an online decision support system for acute coronary syndromes. Circ Cardiovasc Qual Outcomes 2010;3:694-700.

6 Sorajja P, Gersh B], Cox DA et al. Impact of delay to angioplasty in patients with acute coronary syndromes undergoing invasive management: analysis from the ACUITY (Acute Catheterization and Urgent Intervention Triage strategY) trial. J Am Coll Cardiol 2010;55:1416-24.

7 Mehta SR, Granger CB, Boden WE et al. Early versus delayed invasive intervention in acute coronary syndromes. NEJM 2009;360:2165-75.

8 Reichlin T, Schindler C, Drexler B et al. One-hour rule-out and rulein of acute myocardial infarction using high-sensitivity cardiac troponin t. Arch Intern Med 2012;172:1211.

9 Reichlin T, Hochholzer W, Bassetti S et al. Early diagnosis of myocardial infarction with sensitive cardiac troponin assays. $N$ Engl J Med 2009;361:858-67.

10 Roffi M, Patrono C, Collet JP et al. 2015 ESC Guidelines for the management of acute coronary syndromes in patients presenting without persistent ST-segment elevation. Eur Heart J 2016;37:267-315.

11 Pickering JW, Than MP, Cullen L et al. Rapid rule-out of acute myocardial infarction with a single high-sensitivity cardiac troponin $\mathrm{t}$ measurement below the limit of detection. Ann Intern Med 2017;166:715

12 Al-Saleh A, Alazzoni A, Al Shalash S et al. Performance of the highsensitivity troponin assay in diagnosing acute myocardial infarction: systematic review and meta-analysis. C Open 2014;2:E199-207.

13 National Institute for Health and Care Excellence. Myocardial infarction (acute): Early rule out using high-sensitivity troponin tests (Elecsys Troponin T high-sensitive, ARCHITECT STAT High Sensitive Troponin-I and AccuTnI+3 assays). Diagnostics guidance [DG15]. NICE, 2014. 
14 Krintus M, Kozinski M, Boudry P et al. European multicenter analytical evaluation of the Abbott ARCHITECT STAT high sensitive troponin I immunoassay. Clin Chem Lab Med 2014;0:1657-65.

15 Shah ASV, Anand A, Sandoval Y et al. High-sensitivity cardiac troponin I at presentation in patients with suspected acute coronary syndrome: a cohort study. Lancet 2015;386:2481-8.

16 Body R, Carley S, McDowell G et al. Rapid exclusion of acute myocardial infarction in patients with undetectable troponin using a high-sensitivity assay. J Am Coll Cardiol 2011:58:1332-9.

17 NHS Improvement. Reference costs 2016/17: highlights, analysis and introduction to the data. NHS, 2017.

18 Keller T, Zeller T, Ojeda F et al. Serial changes in highly sensitive troponin I assay and early diagnosis of myocardial infarction. JAMA 2011;306:2684-93.
19 Carlton EW, Pickering JW, Greenslade ] et al. Assessment of the 2016 National Institute for Health and Care Excellence high-sensitivity troponin rule-out strategy. Heart 2018;104:665-72.

20 Khan NA, Daskalopoulou SS, Karp I et al. Sex differences in acute coronary syndrome symptom presentation in young patients. JAMA Intern Med 2013;173:1863-71.

21 Slagman A, von Recum J, Möckel M et al. Diagnostic performance of a high-sensitive troponin $\mathrm{T}$ assay and a troponin $\mathrm{T}$ point of care assay in the clinical routine of an Emergency Department: A clinical cohort study. Int J Cardiol 2017;230:454-60.

Address for correspondence: Dr Mark Sweeney, Imperial College Healthcare NHS Trust, Du Cane Road, London W12 OHS, UK.

Email: mark.sweeney1@nhs.net

\section{Are you involved in planning medical services?}

Medical Care is the online evolution of the well-known RCP publication Consultant physicians working with patients. Written by leading medical specialty experts, it is a comprehensive web-based resource for the efficient and effective design of medical services.
Medical Care has been designed to help those involved in the planning and provision of medical services to get a clearer picture of the specialty services that need to be in place to provide great patient care.

The resource looks in detail at the services delivered by 29 medical specialties.

It also includes overarching themes covering the development of physicians and their teams. 